Welcome to the December Edition of the Journal. We have as a new initiative produced this 'extra' edition online only to help get papers into a compiled issue quicker and - in the light of this month's COP26 summit in Glasgow - to reduce environmental impact. Of course, all FG papers are available online and fully citable as soon as they complete the Production process (usually within 3 weeks of acceptance).

I hope very much you like it and enjoy the excellent content.

I have highlighted just some of the papers.

\section{Foreign body ingestion: dos and don'ts}

This is a really helpful and practical guide. Foreign body ingestion - true foreign body ingestion (more common in children) and food bolus impaction - is common. The practical management is not always straightforward. In this issue Becq and colleagues provide authoritative guidance for the management of foreign bodies located in the upper gastrointestinal tract. It is useful to consider true foreign body ingestion and food bolus impaction (where there may be an underlying oesophageal abnormality such as Eosinophilic Oesophagitis) separately. The initial evaluation is important. Most patients are asymptomatic unless the foreign body is stuck. Radiology should not delay urgent treatment. Endoscopic extraction is successful in up to $95 \%$ of cases. There are numerous specifics discussed in the article - sharp and pointed objects, long or bulky objects, coins, magnets, batteries, drugs and food - with specific guidance. There is a useful algorithm and a helpful summary of do's and don'ts. Great article and well worth having to hand when foreign body ingestion present (see page 664).

\section{Eosinophilic oesophagitis: recent advances and practical management}

Eosinophilic Oesophagitis is increasingly recognised (first described in 1993, now one of the the most common causes of oesophageal disease in young people). In this issue Attwood and Epstein review the recent advances and practical management starting with the emotive comment ...the diagnosis everyone needs to know about for the 2000's and we are still learning about this condition. The main symptom is dysphagia.
Presentation can be with food bolus obstruction. Diagnosis is by endoscopy with three level biopsies. Histology is characterised by a dense eosinophilic infiltrate on a background of hyperplastic mucosa. There are multiple different treatment options including dietary restriction, proton pump inhibitors and local steroid therapy. Refractory cases need specialist assessment. Strictures can occur in up to $10 \%$. This review provides an excellent summary of what we know and how best to manage based on current knowledge. Essential reading. Listen to the accompanying podcast. Editor's choice this month (see page 644).

\section{British Intestinal Failure Alliance (BIFA) guidance - haematological and biochemical monitoring of adult patients receiving home parenteral nutrition}

This is an important paper in which the authors (on behalf of the British Intestinal Failure Alliance) collate the existing guidance - British Association of Parenteral and Enteral Nutrition, European Society of Parenteral and Enteral Nutrition, National Institute of Health and Care excellent and the Australasian Society of Parenteral and Enteral Nutrition - into a clear and concise review. Most of the recommendations are consensus rather than evidence based reflecting the paucity of highquality evidence. There are useful tables summarising the recommendations which can easily be adopted into clinical practice - the different guidelines are compared and there is a useful discussion of each of the different parameters measured including factors that should be considered when interpreting results. This is an invaluable resource for clinicians - read the paper and linked podcast. Editor's Choice this month (see page 656).

\section{Important relation between self-efficacy, sense of coherence, illness perceptions, depression, and anxiety in patients with inflammatory bowel disease}

Anxiety and depression are common in inflammatory bowel disease and we are all aware have a significant impact on the disease course. In this issues EindorAbarbanel and colleagues investigate the association of illness perception (IP), self-efficacy (SE) and sense of coherence (SE) with anxiety and depression in patients with IBD - 299 patients, median age 34 years. There is a useful discussion of the terminology in the paper. In brief Illness perception is an individual's mental representation of a disease; Sense of coherence is a theoretical construct developed to explain why some people, regardless of stressful events, fall ill and others do not; Self-efficacy is the belief that an individual can carry out a task necessary to achieve a desired outcome. Depression and anxiety were assessed using the Hospital Anxiety and Depression Scale. IP, SE and SOC were assessed using the Brief Illness perception Questionnaire, IBD-SE and SOC scales. Lower results in IP, SE and SOC were found to be associated with significantly increased anxiety (OR 8.35, p <0.001; OR 4.18, p=0.001; OR 4.67, $\mathrm{p}<0.001$, respectively) and depression (OR 15.8, p=0.001; OR 10.99, $\mathrm{p}=0.029$; OR 6.12, $\mathrm{p}=0.014$ ) on multivariate analysis. IBD is a complex disorder with significant morbidity and these data further support the necessary increase in psychological support for patients to assess, consider and intervene where possible with the likely outcome that this will improve treatment efficacy and quality of life (see page 601).

\section{Gut-focused hypnotherapy for children and adolescents with irritable bowel syndrome}

Irritable Bowel Syndrome is common in children and adolescents with a significant potential impact on quality of life, education and socialisation. The aetiology is multifactorial and treatment options are limited. In this issue Vasant and colleagues report their 'real world' experience with Gut-focussed hypnotherapy -32 patients, median age 16 years, $72 \%$ female - with severe IBS as per the ROME 111 diagnostic criteria. All had 12 sessions of GFH. 28/32 responded with significant reduction in the mean IBS symptom severity score (IBS-SSS). Non-colonic symptoms, anxiety and depression scores and quality of life also improved. The report confirms treatment efficacy - clearly more research is needed - but from this report Gut focused hypnotherapy is an appropriate treatment option in children and adolescents with IBS and we should actively 
consider accessing it for our patients (see page 570).

\section{Functional abdominal pain in adolescents: case-based management}

Functional gastrointestinal disorders (FGIDs), including functional abdominal pain (FAP), account for a large portion of conditions seen by paediatricians. Use of the biopsychosocial model (biological, psychological, social) is essential in conceptualising, evaluating and treating cases. In this article Yacob and colleagues review the assessment and management with specific focus on FAP in adolescents using a case-based approach. The case vignette will be familiar to clinicians. Pathophysiology is multifactorial and not always straightforward. It is important to be confident in the diagnosis and avoid over investigation. Treatment options are considered in detail (including the limited evidence base). Functional Gastrointestinal disorders and in particular the pain predominant have significant impact on the child's quality of life and ability to function. It well recognised that children and families respond best when clinicians take the problem seriously, listen carefully, explain the diagnosis thoroughly and present a comprehensive therapeutic plan (see page 629).

\section{Telephone clinic improves gluten-free dietary adherence in adults with coeliac disease: sustained at 6 months}

Coeliac disease (CD) is common. Treatment is with a gluten free (GF) diet and non-adherence is associated with increased morbidity. The difficult question is how to best help such cases, particularly as we recover from the pandemic with the greater potential for remote rather than face to face consultations. In this issue Muhammad and colleagues report on the impact of a personalised telephone clinic (125 patients, 30 non adherent). Validated questionnaires were used to assess GF dietary adherence (Coeliac Disease Adherence Test; CDAT), knowledge of GF foods and CD-related quality of life (QoL). GF dietary adherence (CDAT) median scores significantly improved at 3 and 6 months after the telephone clinic compared with baseline, $\mathrm{p}<0.01)$. Change in GF dietary knowledge score was associated with improved GF dietary adherence CDAT score $(r=-0.22 ; p=0.039)$. In summary the clinic has a positive impact on dietary knowledge and GF dietary adherence for targeted individuals and although we are limited by resources to deliver such initiatives needs the intervention should be considered because of the potential long-term impact on disease control and quality of life.

There is an excellent accompanying commentary - Call me maybe? Telephone clinics for coeliac disease dietetic services - which highlights the paucity of evidence we have to inform quality criteria for CD dietetic care and calls more high-quality multidisciplinary research like this, driven by dietitians, physicians, and patients, to inform how we deliver the cornerstone of CD management: the gluten free diet (see page 586).

I hope very much you enjoy this bumper edition. Please access the journal website for the latest content

Please continue to read, enjoy, and feedback on the journal. Follow us on twitter $@$ FrontGastro_BMJ and listen to our regular podcasts accessed via the journal website https://fg.bmj.com/

ORCID iD

R Mark Beattie http://orcid.org/00000003-4721-0577 